Article

\title{
Security Analysis of Multi-Antenna NOMA Networks Under I/Q Imbalance
}

\author{
Xingwang Li ${ }^{1}$, Mengle Zhao ${ }^{1}{ }^{\oplus}$, Changsen Zhang ${ }^{1}$, Wali Ullah Khan ${ }^{2}{ }^{\oplus}$, Jun $\mathrm{Wu}^{1, *}$, \\ Khaled M. Rabie ${ }^{3}$ and Rupak Kharel ${ }^{4}$ (D) \\ 1 School of Physics and Electronic Information Engineering, Henan Polytechnic University, \\ Jiaozuo 454003, China; lixingwangbupt@gmail.com (X.L.); zhaomenglehpu@163.com (M.Z.); \\ zhangchangsen@hpu.edu.cn (C.Z.) \\ 2 School of Information Science and Engineering, Shandong University, Qingdao 266237, China; \\ waliullahkhan30@gmail.com \\ 3 Faculty of Science and Engineering, Manchester Metropolitan University, Manchester M1 5GD, UK; \\ K.Rabie@mmu.ac.uk \\ 4 Department of Computing and Mathematics, Manchester Metropolitan University, \\ Manchester M15 6BH, UK; R.Kharel@mmu.ac.uk \\ * Correspondence: wujun@hpu.edu.cn; Tel.: +86-159-3911-8263
}

Received: 14 October 2019; Accepted: 7 November 2019; Published: 11 November 2019

\begin{abstract}
This paper investigates the reliability and security performance of the downlink non-orthogonal multiple access (NOMA) networks over Nakagami- $m$ fading channels, where the base station (BS) aims to communicate with multi-antenna NOMA users in the presence of a multi-antenna eavesdropper. To be more practical, a detrimental factor at both transmitter and receiver is considered, namely in-phase and quadrature-phase imbalance (IQI). To further improve the reliability and security of the considered networks, the selection combining (SC) algorithm at the receiver is taken into account. More specifically, the exact analytical expressions for the outage probability (OP) and the intercept probability (IP) are derived in closed-form. To obtain a better understanding of the influence for the IQI parameters on the system performance, the asymptotic behaviors for the outage probabilities (OPs) in the high signal-to-noise ratio (SNR) region are analyzed. Based on the asymptotic results, the diversity order of the considered system are obtained and discussed. The numerical results are presented to verify the validity of the theoretical analysis.
\end{abstract}

Keywords: non-orthogonal multiple access; in-phase and quadrature-phase imbalance; physical layer security; selection combining

\section{Introduction}

Non-orthogonal multiple access (NOMA) is widely known as one of the important multiple access technologies in the future wireless communication systems, which can achieve high spectral efficiency, massive connection and low delay [1]. In general, NOMA technologies are typically classified into three categories, namely, power-domain NOMA, sparse code multiple access (SCMA), and pattern division multiple access (PDMA) [2]. Compared with the traditional orthogonal multiple access (OMA), the main characteristic of NOMA is that it allows different users to occupy the same frequency spectrum, time and space resources by power-domain multiplexing [3]. At the transmitter, all the information is processed according to superposition coding, and then the successive interference cancellation (SIC) is carried out at the receiver to extract its own information [4]. Apart from the above aspects, NOMA can also ensure the fairness of users by allocating more power to users with weak channel conditions [5]. 
NOMA networks has been extensively studied in wireless communication [6-11]. In [6], the authors evaluated the performance of downlink NOMA networks over different modulation schemes and analyzed the impact of system parameters on the system performance. To provide high diversity and extend the coverage of network, cooperative communication has been introduced into NOMA networks, and the analytical closed-form expressions for the outage probability (OP) and the ergodic sum rate (ESR) by using amplify-and-forward (AF) relaying were derived [7]. In [8], the outage performance and asymptotic behaviors for NOMA and OMA networks over Nakagami- $m$ fading channels was explored, which demonstrated that NOMA can provide better fairness than the traditional OMA. In addition, the outage performance of the NOMA-based downlink cooperative relay networks was studied in [9], which also showed that NOMA can provide better fairness and higher spectral efficiency. Furthermore, NOMA can also be applied to millimeter-wave (mmWave) systems [10] and wireless cognitive radio (CR) networks [11].

Unfortunately, the above research works are basically based on the ideal radio-frequency (RF) front-end. In the actual communication systems, due to the mismatch of analog components and the non-ideal transceiver manufacturing, it is easy to lead to defects in the RF front-end, thus limiting the overall performance of the system. In this paper, the main studied in-phase and quadrature-phase imbalance (IQI) is an important factor of these defects, which refers to the mismatch between the amplitude/phase of the in-phase and quadrature-phase of the signals at the transceiver. It leads to the imperfection of image suppression, and finally leads to the degradation of system performance [12]. Specifically, the performance of the AF dual-hop relaying systems in the presence of IQI was studied by deriving the exact expressions for the OP and ergodic capacity of the considered systems in [13]. The authors of [14] investigated the effect of RF front-end IQI based on space time block coding (STBC)orthogonal frequency division multiplexing (OFDM) systems. Similarly, the authors analyzed the effects of IQI and Phase noise (PHN) on the beamforming of OFDM direct-conversion transceivers in [15]. Several research works have been proposed to compensate for the impact of IQI schemes and algorithms [16-18]. In [16], the authors proposed a finite-impulse-response (FIR) structure to compensate IQI. A method of modifying the transmission channel IQI was proposed in [17]. Based on the least mean square (LMS) and recursive least square (RLS) adaption algorithms, the authors proposed two blind adaptive IQI compensation algorithms to improve the image rejections [18]. However, only a few research works studied the impact of IQI on the performance of NOMA-based wireless communication systems, which discussed the OP of the single-carrier and multi-carrier and proved that IQI would have a different effect on each user of NOMA-based system [19].

Since the broadcast characteristic of wireless transmission, this makes wireless communication systems vulnerably attack by illegal users, thus, it is vital to ensure the security of the communication networks [20]. The traditional encryption mechanism protects the information transmission by adopting the encryption algorithms, which rely on complexity and time-consumption [21]. Different from the network layer security, the physical layer security (PLS) is to consider the security of wireless networks from the aspects of information theory [22]. The dominant feature of PLS is to utilize the time-varying nature of the wireless fading channel to guarantee reliable communication [23]. A PLS scheme based on channel pre-compensation was proposed in [24], which improved the security performance of the system. The authors of [25] proposed a hybrid cooperative beamforming and interference scheme, which proved that it can effectively improve the transmission security of physical layer networks in the presence of eavesdropper. In [26], the authors investigated the average secret capacity (ASC) and the secure outage probability (SOP) over $\alpha-\eta-\kappa-\mu$ fading channels. In order to improve the security of the system, the authors proposed a transmit beamforming scheme to maximize the signal-to-noise ratio (SNR) of the primary receivers, and analyzed the secure performance of the system [27]. Therefore, PLS technology plays an important role in guaranteeing the security of the wireless communication systems.

Recently, the security of NOMA systems has been extensively investigated [28-33]. The secure outage performance of the NOMA system was investigated under two connections: (1) Direct 
transmission phase; (2) Cooperation phase [28], in which considered the base station (BS) has the perfect channel state information (CSI) of each legitimate user and unknown the CSI of the eavesdropper. In [29], the authors proposed a best-secure-near-user best-secure-far-user (BSNBSF) scheme to improve the secure performance of multi-user NOMA networks. To improve the security of NOMA users, the authors presented a scheme of using media access control (MAC) address and international mobile equipment identity (IMEI) [30]. In addition, the authors of [31] designed and analyzed the secure transmission in full-duplex (FD) two-way relay networks (TWRN) with artificial noise in the presence of eavesdropping. The security performance of the multiple eavesdroppers system with collusion and non-collusion eavesdroppers scenarios was studied [32]. The closed-form expressions of SOP and strictly positive secrecy capacity (SPSC) for cooperative NOMA systems over Rayleigh fading channels were derived in [33].

Multi-antenna is another physical layer technology to improve the performance of a wireless communication system. By introducing multi-antenna into the NOMA systems, it can achieve the advantages of multi-antenna and NOMA. Therefore, some research works have investigated the combined technology; see [34-38] and the references therein. Authors in [34] studied the performance of multiple-antenna NOMA and OMA, and it indicated that the performance of multiple-antenna NOMA outperforms that of multiple-antenna OMA one. The antenna selection (AS) technology has the advantages of low cost, low complexity, high diversity gains, which can solve the computational complexity and power consumption issues caused by multiple antennas. Therefore, the combination of NOMA and AS has attracted significant attention [35-38]. In [35], the authors analyzed the secure outage performance of MISO-NOMA systems by deriving the exact analytical expressions of SOP under optimal antenna selection (OAS) and suboptimal antenna selection (SAS) schemes, and proposed an effective power allocation scheme to obtain the non-zero diversity order under the two AS schemes. The authors analyzed the security performance of the uplink NOMA system, where the BS is equipped with multiple antennas [36]. Regarding hardware impairments at transceiver, the authors of [38] investigated the reliability and security of multiple-antenna cooperative networks by proposing three SC schemes [37]. The most widely used is SC where the destination selects a branch with the highest SNR to be connected to the output portion of the receiver. In this paper, we adopt SC scheme since it outputs the signals of the branch with the largest signal-to-interference-plus-noise ratio (SINR) and low complexity [38].

Although a great number of works have studied the influence of HIs on NOMA-based systems, to the best of our knowledge, there is no open literature to analyze the impact of IQI on the PLS performance of NOMA-based systems. Motivated by the aforementioned discussions, we study the reliability and security of NOMA system in terms of OP and IP in the presence of IQI. In order to analyze the effects of IQI and system parameters on the performance of the considered system, we derive the asymptotic OP in the high SNR region and discuss asymptotic behavior in terms of diversity order. In addition, the Nakagami- $m$ fading channels is considered with the following reasons (Lately, some research contributions have investigated the PLS over generalized fading channels under ideal conditions [39,40], such as $\alpha-\mu, \kappa-\mu$ etc. These fading channels extensively characterize some realistic propagation environments. Therefore, studying the security performance of the wireless communication systems over these fading channels in the presence of IQI will constitute our future research work.): (i) It can be used to capture the distribution characteristics of the received signal of both diffuse and specular scattering; (ii) It can be applied to many types of propagation environments, such Gaussian, line-of-sight (LoS) and non-LoS (NLoS); (iii) It provides a mathematical tractable for the system performance evaluation. The primary contributions of our paper are summarized as follows:

- Considering IQI at both transmitter (TX) and receiver (RX), we propose an AS scheme at the multiple-antenna legitimate users and eavesdropper to enhance reliability and security, in which the antenna of optimal quality link between the source and the receiver antennas is selected.

- Based on the proposed selection scheme, we derive the exact analytical expressions for the OP and intercept probability (IP) of the considered single-input multiple-output (SIMO)-NOMA system 
over the Nakagami- $m$ fading channels. The results show that the reliability of the considered system can be improved by increasing the number of antennas at the receiver, while the security of the system will be reduced with the increase of the number of antennas at the eavesdropper.

- In order to obtain deeper insights, we investigate the asymptotic behaviors of OP in the high SNR regime. Based on the derived asymptotic results, the diversity order for the OP can be derived and discussed. Specifically, it is proved that there is a trade-off between OP and IP. Moreover, considering the different degrees of impairment, the diversity order of the asymptotic OP for the considered system is derived, and the results demonstrate that IQI does not affect the diversity order of the considered system.

The rest of this paper is organized as follows: Section 2 presents the SIMO-NOMA system and channel model in the presence of IQI. In Section 3, we derive the exact closed-form expressions for the OP and IP of the considered system in order to investigate the reliability and security. Section 4 provides some numerical results and discussions, while conclusion of this paper is given in Section 5 .

Notation: In this paper, the main notations are shown as follows: the $E\{\cdot\}$ and $|\cdot|$ denote the expection and absolute operations, respectively. A complex Gaussian random variable with mean $\mu$ and variance $\sigma^{2}$ reads as $\mathcal{C N}\left\{\mu, \sigma^{2}\right\} .(\cdot)^{*}$ denotes conjugation. $\Re\{x\}$ and $\Im\{x\}$ represent the real and imaginary parts of $x$, respectively. Notation $\operatorname{Pr}(\cdot)$ is the probability, $\Gamma(\cdot)$ denotes the Gamma function, $\mathrm{X}$ ! denotes the factorial of $\mathrm{X}$, while $f_{x}(\cdot)$ and $F_{x}(\cdot)$ are the probability density function (PDF) and the cumulative distribution function $(\mathrm{CDF})$ of a random variable, respectively.

\section{System Model}

We consider a SIMO-NOMA downlink system, as depicted in Figure 1, which consists of one source $S, N$ legitimate destinations $\left(D_{1}, D_{2} \cdots D_{N}\right)$, one eavesdropper $(E)$. It is assumed that, each $D$ and $E$ are respectively equipped with $M$ antennas and $K$ antennas, while $S$ is equipped with a single antenna. Without loss of generality, the channel gains of between $S$ and $D$ are ordered as $\left|h_{S D_{1, m}}\right|^{2}<\ldots<\left|h_{S D_{n, m}}\right|^{2}<\ldots<\left|h_{S D_{N, m}}\right|^{2}$. We assume that both the legitimate and the eavesdropping channels are subject to independent and non-identically distributed Nakagami- $m$ distributions. The channel gain $\rho_{i}$ follows the Gamma distribution with $\rho_{i}=\left|h_{i}\right|^{2} \sim \operatorname{Gamma}\left(\alpha_{i}, \beta_{i}\right)$. Thus, the PDF and CDF of the unsorted channel gain $\rho_{i}$ are respectively expressed as [41]

$$
\begin{gathered}
f_{\rho_{i}}(x)=\frac{x^{\alpha_{i}-1}}{\Gamma\left(\alpha_{i}\right) \beta_{i}{ }^{\alpha_{i}}} e^{-\frac{x}{\beta_{i}}}, \\
F_{\rho_{i}}(x)=1-\sum_{g_{i}=0}^{\alpha_{i}-1} \frac{e^{-\frac{x}{\beta_{i}}}}{g_{i} !}\left(\frac{x}{\beta_{i}}\right)^{g_{i}},
\end{gathered}
$$

where $\alpha_{i} \geq 1$ and $\beta_{i}>0$ are the shape and scale parameters of Gamma function, respectively. With the aid of order statistics, the PDF and CDF of the order channel gains $\rho_{s d_{n, m}}$ are represented as [42]

$$
\begin{gathered}
f_{\rho_{s d_{n, m}}}(x)=\frac{N !}{(n-1) !(N-n) !} f_{\rho_{i}}(x)\left[F_{\rho_{i}}(x)\right]^{n-1}\left[1-F_{\rho_{i}}(x)\right]^{N-n}, \\
F_{\rho_{s d_{n, m}}}(x)=\frac{N !}{(n-1) !(N-n) !} \sum_{l=0}^{N-n}\left({ }^{N-n}\right) \frac{(-1)^{l}}{n+l}\left[F_{\rho_{i}}(x)\right]^{n+l} .
\end{gathered}
$$

To be more practical, we assume that both TX and RX are subject to IQI, in which case the transmitted baseband with IQI can be expressed as

$$
x_{s}=\mu_{t} y_{s}+v_{t}\left(y_{s}\right)^{*},
$$

where $y_{s}=\sum_{n=1}^{N} \sqrt{a_{n} P_{S}} x_{n}$ is the baseband transmitted signal under ideal I/Q with $E\left\{\left|x_{n}\right|^{2}\right\}=1$; $P_{S}$ is the average transmit power of signal $S ; x_{n}$ is the signal of the $n$-th sorted user. Also, $a_{n}$ is the 
power allocation coefficient of the $n$-th sorted user, which satisfies $a_{1} \geq \cdots \geq a_{N}$ and $\sum_{n=1}^{N} a_{n}=1$. $\mu_{t}=\left(1+\varsigma_{t} \exp \left(j \phi_{t}\right)\right) / 2, v_{t}=\left(1-\varsigma_{t} \exp \left(j \phi_{t}\right)\right) / 2$, where $\varsigma_{t}$ and $\phi_{t}$ are the amplitude and phase mismatch levels at TX. Considering IQI at the RX, the received signals at $D_{n}$ and $E$ are respectively given by

$$
\begin{gathered}
y_{D_{n}}=\mu_{r_{n}}\left(h_{S D_{n, m}} x_{S}+n_{0}\right)+v_{r_{n}}\left(h_{S D_{n, m}} x_{s}+n_{0}\right)^{*}, \\
y_{E}=\mu_{r_{e}}\left(h_{S E_{k}} x_{S}+n_{0}\right)+v_{r_{e}}\left(h_{S E_{k}} x_{S}+n_{0}\right)^{*},
\end{gathered}
$$

where both $h_{S D_{n, m}}$ and $h_{S E_{k}}$ denote the channel coefficients $S \rightarrow D_{n, m}$ and $S \rightarrow E_{k}$, respectively; $n_{0} \sim \mathcal{C N}\left(0, N_{0}\right)$ represents the complex additive white Gaussian noise (AWGN); $\mu_{r_{n}}, v_{r_{n}}$ and $\mu_{r_{e}}, v_{r_{e}}$ correspond to the IQI parameters of the $n$-th user and $E$, respectively. $\mu_{r_{j}}=\left(1+\varsigma_{r_{j}} \exp \left(j \phi_{r_{j}}\right)\right) / 2$, $v_{r_{j}}=\left(1-\varsigma_{r_{j}} \exp \left(j \phi_{r_{j}}\right)\right) / 2, j \in\{n, e\}$, where $\varsigma_{r_{j}}$ and $\phi_{r_{j}}$ are the amplitude and phase mismatch levels at RX, the typical ranges of $\varsigma_{t}, \varsigma_{r_{j}}$ and $\phi_{t}, \phi_{r_{j}}$ are $1 \sim 1.5$ and $0^{\circ} \sim 30^{\circ}$, respectively [43]. It should be noted that for ideal RF front end, the IQI parameters are $\zeta_{t}=\varsigma_{r_{j}}=1.0, \phi_{t}=\phi_{r_{j}}=0^{\circ}$, which correspond to $\mu_{t}=\mu_{r_{j}}=1$ and $v_{t}=v_{r_{j}}=0$. The severity of TX IQI and RX IQI can be determined by the TX/RX image rejection ratio (IRR), which is defined as [44]

$$
I R R_{t / r_{j}}=\frac{\left|\mu_{t / r_{j}}\right|^{2}}{\left|v_{t / r_{j}}\right|^{2}} .
$$

According to the NOMA protocol, perfect SIC is performed, and we can obtain

$$
\begin{aligned}
y_{D_{n}} & =\left(\left(\mu_{r_{n}} \mu_{t}-1\right)+v_{r_{n}} v_{t}^{*}\right) h_{S D_{n, m}} \sum_{i=1}^{n-1} \sqrt{a_{i} P_{s}} x_{i}+\left(\mu_{r_{n}} \mu_{t}+v_{r_{n}} v_{t}^{*}\right) h_{S D_{n, m}} \sum_{i=n}^{N} \sqrt{a_{i} P_{s}} x_{i} \\
& +\left(\mu_{r_{n}} v_{t}+v_{r_{n}} \mu_{t}^{*}\right) h_{S D_{n, m}} \sum_{i=1}^{N} \sqrt{a_{i} P_{s}} x_{i}^{*}+\mu_{r_{n}} n_{0}+v_{r_{n}} n_{0}, \\
y_{E} & =\left(\left(\mu_{r_{e}} \mu_{t}-1\right)+v_{r_{e}} v_{t}^{*}\right) h_{E} \sum_{i=1}^{n-1} \sqrt{a_{i} P_{s}} x_{i}+\left(\mu_{r_{e}} \mu_{t}+v_{r_{e}} v_{t}^{*}\right) h_{E} \sum_{i=n}^{N} \sqrt{a_{i} P_{s}} x_{i} \\
& +\left(\mu_{r_{e}} v_{t}+v_{r_{e}} \mu_{t}^{*}\right) h_{E} \sum_{i=1}^{N} \sqrt{a_{i} P_{s}} x_{i}^{*}+\mu_{r_{e}} n_{0}+v_{r_{e}} n_{0} .
\end{aligned}
$$

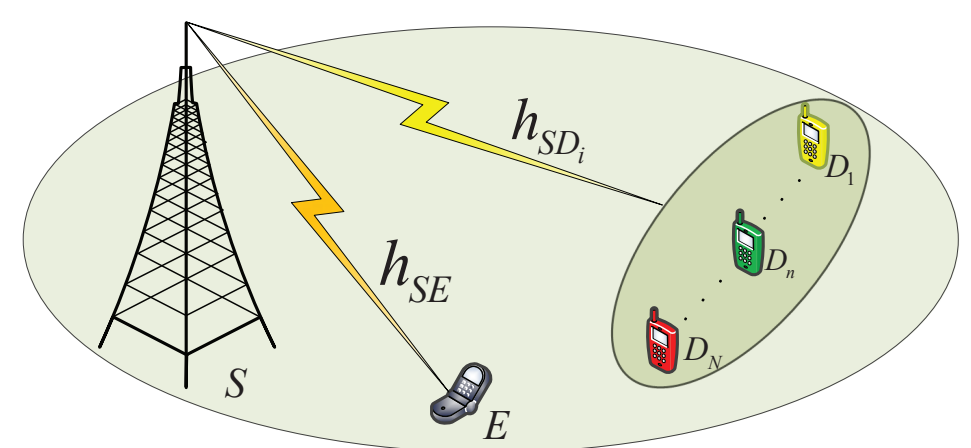

Figure 1. Single-input multiple-output (SIMO)-non-orthogonal multiple access (NOMA) system model with eavesdropper.

Since $D_{n}$ and $E$ are equipped with multiple antennas, we adopt SC to detect the received signals at receiver (In this study, we consider SC scheme, but the results herein can be extended to other 
receiver schemes as well, such as maximal ratio combing (MRC) and equal gain combination (EGC).). Then, one optimal antenna $\widehat{m}$ is selected according to the SNR of the $S$ to $D_{n}$ as

$$
\widehat{m}=\arg \max _{m=1,2, \ldots M} \rho_{S D_{n, m}}
$$

one optimal antenna $\widehat{k}$ is selected according to the SNR of the $S$ to $E$ as

$$
\widehat{k}=\arg \max _{k=1,2, \ldots K} \rho_{S E_{k}} .
$$

Therefore, the SINR at the output for the $D_{n}$ to decode the $D_{p}$ 's information $x_{p}(p \leq n)$, can be written as

$$
\gamma_{p \rightarrow n}^{S D}=\frac{\rho_{S D_{n, \hat{m}}} \gamma A_{n} a_{p}}{\rho_{S D_{n, \hat{m}}} \gamma D_{n} \sum_{i=1}^{p-1} a_{i}+\rho_{S D_{n, \hat{m}}} \gamma A_{n} \sum_{i=p+1}^{N} a_{i}+\rho_{S D_{n, \hat{m}}} \gamma B_{n}+C_{n}}
$$

where $\gamma=P_{S} / N_{0}$ is the SNR at $S, \rho_{S D_{n, \hat{m}}}=\left|h_{S D_{n, \hat{m}}}\right|^{2} . A_{n}=\left|\mu_{r_{n}} \mu_{t}+v_{r_{n}} v_{t}^{*}\right|^{2}=\left|\mu_{r_{n}} \mu_{t}\right|^{2}+\left|v_{r_{n}} v_{t}^{*}\right|^{2}+$ $2 \Re\left\{\mu_{r_{n}} \mu_{t} v_{r_{n}} v_{t}^{*}\right\}, B_{n}=\left|\mu_{r_{n}} v_{t}+v_{r_{n}} \mu_{t}^{*}\right|^{2}=\left|\mu_{r_{n}} v_{t}\right|^{2}+\left|v_{r_{n}} \mu_{t}^{*}\right|^{2}+2 \Re\left\{\mu_{r_{n}} v_{t} v_{r_{n}} \mu_{t}^{*}\right\}, C_{n}=\left|\mu_{r_{n}}\right|^{2}+\left|v_{r_{n}}\right|^{2}$, $D_{n}=\left|\mu_{r_{n}} \mu_{t}-1\right|^{2}+\left|v_{r_{n}} v_{t}\right|^{2}$.

For direct conversion transceivers, IRR is generally in the range of 20-40 $\mathrm{dB}$ [45], whereas due to the inequality, it can be safely assumed that [46]

$$
2 \Re\left\{\mu_{r_{n}} \mu_{t} v_{r_{n}} v_{t}^{*}\right\} \ll\left|\mu_{r_{n}} \mu_{t}\right|^{2}+\left|v_{r_{n}} v_{t}^{*}\right|^{2},
$$

and

$$
2 \Re\left\{\mu_{r_{n}} v_{t} v_{r_{n}} \mu_{t}^{*}\right\} \ll\left|\mu_{r_{n}} v_{t}\right|^{2}+\left|v_{r_{n}} \mu_{t}^{*}\right|^{2} .
$$

Therefore, $A_{n}$ and $B_{n}$ can be accurately represented as

$$
\begin{aligned}
& A_{n} \approx\left|\mu_{r_{n}} \mu_{t}\right|^{2}+\left|v_{r_{n}} v_{t}^{*}\right|^{2}, \\
& B_{n} \approx\left|\mu_{r_{n}} v_{t}\right|^{2}+\left|v_{r_{n}} \mu_{t}^{*}\right|^{2} .
\end{aligned}
$$

According to the NOMA principle, when the $D_{n}$ 's signals is eavesdropped, $E$ successfully eliminates the signal of $D_{l}(1<l<n)$ with SIC, whereas the signals of $D_{l}(N \geq l>1)$ are treated as noise. According to (10), the SINR required to eavesdrop the $D_{n}$ 's message at $E$ is given by

$$
\gamma_{S E_{\widehat{k}}}^{n}=\frac{\rho_{S E_{\widehat{k}}} \gamma A_{e} a_{n}}{\rho_{S E_{\widehat{k}}} \gamma D_{e} \sum_{i=1}^{k-1} a_{i}+\rho_{S E_{\widehat{k}}} \gamma A_{e} \sum_{i=k+1}^{N} a_{i}+\rho_{S E_{\widehat{k}}} \gamma B_{e}+C_{e}},
$$

where $\rho_{S E_{\widehat{k}}}=\left|h_{S E_{\hat{k}}}\right|^{2} \cdot A_{e} \approx\left|\mu_{r_{e}} \mu_{t}\right|^{2}+\left|v_{r_{e}} v_{t}^{*}\right|^{2}, B_{e} \approx\left|\mu_{r_{e}} v_{t}\right|^{2}+\left|v_{r_{e}} \mu_{t}^{*}\right|^{2}, C_{e}=\left|\mu_{r_{e}}\right|^{2}+\left|v_{r_{e}}\right|^{2}, D_{e}=$ $\left|\mu_{r_{e}} \mu_{t}-1\right|^{2}+\left|v_{r_{e}} v_{t}\right|^{2}$.

\section{Performance Analysis}

In this section, we evaluate the reliability and security of the considered system by deriving the exact analytical expressions for the OP and IP in closed-form. Moreover, we examine asymptotic outage behavior in the high SNR regime, and then we obtain the diversity order by using the derived asymptotic results. 


\subsection{Outage Probability}

The OP is a way of characterizing the link quality. If the link SINR cannot meet the required threshold, an outage event will occur, which is defined that outage event occurs at $D_{n}$ if it fails to decode its own signal or the signal of $D_{p}$. Therefore, the OP at $D_{n}$ is evaluated as

$$
P_{\text {out }}^{D_{n}}=1-\operatorname{Pr}\left(E_{n, 1}^{S D} \cap \cdots \cap E_{n, n}^{S D}\right)
$$

where $E_{n, p}^{S D}$ denotes $D_{n}$ can successfully decode the signal of $D_{p}$, which can be expressed as

$$
\begin{aligned}
E_{n, p}^{S D} & =\left\{\gamma_{p \rightarrow n}^{S D}>\gamma_{t h p}\right\} \\
& =\left\{\rho_{S D_{n, \hat{m}}}>\theta_{p}\right\},
\end{aligned}
$$

where $\gamma_{\text {thp }}=2^{2 R_{p}}-1$, and $R_{p}$ denotes $D_{p}$ 's target rate, $\theta_{p}=C_{n} \gamma_{t h p} /\left[\gamma\left(A_{n} a_{p}-\gamma_{t h p} D_{n} \sum_{i=1}^{p-1} a_{i}-\gamma_{t h p} A_{n} \sum_{i=p+1}^{N} a_{i}-\gamma_{t h p} B_{n}\right)\right]$.

Utilizing the above discussion, the exact closed-form expressions for the $\mathrm{OP}$ of the considered system over Nakagami- $m$ fading channels in the presence of IQI are provided in the following theorem.

Theorem 1. The exact closed-form expressions for the OP of $D_{n}$ can be expressed as

- Non-ideal conditions

$$
\begin{gathered}
P_{o u t}^{D_{n}, n i}=\frac{N !}{(n-1) !(N-n) !} \sum_{l=0}^{N-n}\left(N_{l}^{N-n}\right) \frac{(-1)^{l}}{n+l} \sum_{b=0}^{M(n+l)} U_{b}(-1)^{b} U_{b} V_{b}\left(\widehat{\theta}_{n}\right)^{\bar{b}} e^{-\frac{\hat{\theta}_{n}}{\beta_{i}}}, \\
\text { for } 0<\gamma_{\text {thp }}<\frac{A_{n} a_{p}}{D_{p} \sum_{i=1}^{p-1} a_{i}+A_{n} \sum_{i=p+1}^{N} a_{i}+B_{n}} \text {, and } P_{\text {out }}^{D_{n}, n i}=1 \text { for } \gamma_{\text {thp }}>\frac{A_{n} a_{p}}{D_{p} \sum_{i=1}^{p-1} a_{i}+A_{n} \sum_{i=p+1}^{N} a_{i}+B_{n}} .
\end{gathered}
$$

- Ideal conditions $\left(\varsigma_{t}=\varsigma_{r_{j}}=1.0, \phi_{t}=\phi_{r_{j}}=0^{\circ}\right)$

$$
\begin{gathered}
P_{\text {out }}^{D_{n}, i d}=\frac{N !}{(n-1) !(N-n) !} \sum_{l=0}^{N-n}\left(\begin{array}{l}
N-n \\
l
\end{array}\right) \frac{(-1)^{l}}{n+l} \sum_{b=0}^{M(n+l)} U_{b} U(-1)^{b} U_{b} V_{b}(\theta)^{\bar{b}} e^{-\frac{\theta}{\beta_{i}} b}, \\
\text { for } 0<\gamma_{\text {thp }}<\frac{a_{p}}{\sum_{i=p+1}^{N} a_{i}} \text {, and } P_{\text {out }}^{D_{n}, i d}=1 \text { for } \gamma_{\text {thp }}>\frac{a_{p}}{\sum_{i=p+1}^{N} a_{i}}
\end{gathered}
$$

where

$$
\begin{gathered}
\hat{\theta}_{n}=\max _{1 \leq p \leq n} \theta_{p}, \\
\theta=\max _{1 \leq p \leq n} \frac{\gamma_{t h p}}{\gamma\left(a_{p}-\gamma_{t h p} \sum_{i=p+1}^{N} a_{i}\right)}, \\
\bigcup_{b} \triangleq \sum_{q_{1}} \sum_{q_{2}=0}^{q-q_{1}} \ldots \sum_{q_{\alpha_{i}-1}=0}^{q-q 1-\ldots q_{\alpha_{i}-2}}, \\
V_{q}=\left(\frac{1}{\left(\alpha_{i}-1\right) !}\left(\frac{1}{\beta_{i}}\right)^{M(n+l)}\right)\left(\begin{array}{l}
q \\
q \\
q_{1}
\end{array}\right)\left(\begin{array}{l}
q-q_{1} \\
q_{2}
\end{array}\right) \cdots\left(\begin{array}{l}
q-q_{1}-\cdots-q_{\alpha_{i}-2} \\
q_{\alpha_{i}-1}
\end{array}\right), \\
\bar{q}=\left(\alpha_{i}-1\right)\left(q-q_{1}-\cdots-q_{\alpha_{i}-1} \alpha_{i}-2\right. \\
\prod_{g_{i}=0}^{q}\left(\frac{1}{g_{i} !}\left(\frac{1}{\beta_{i}}\right)^{g_{i}}\right)^{q_{g_{i}+1}},
\end{gathered}
$$


Proof. See Appendix A.

\subsection{Intercept Probability}

In this subsection, we investigate the security of considered system over Nakagami- $m$ fading channels with IQI in terms of IP. The intercept event will occur if the information at $D_{n}$ is successfully eavesdropped by $E$, which is given by

$$
P_{\text {int }}^{n}=\operatorname{Pr}\left(C_{E}^{n}>R_{n}\right),
$$

where $R_{n}$ represents the target rate of $D_{n}$, and according to $C_{E}^{n}=\log _{2}\left(1+\gamma_{S E}^{n}\right)$, (24) can also be expressed as

$$
\begin{aligned}
P_{\text {int }}^{n} & =\operatorname{Pr}\left(\gamma_{S E, \widehat{k}}^{n}>\gamma_{t h e, n}\right) \\
& =\operatorname{Pr}\left(\rho_{S E_{\widehat{k}}}>\delta_{n}\right)
\end{aligned}
$$

where $\gamma_{\text {the }, n}=2^{2 R_{n}}-1, \delta_{n}=C_{e} \gamma_{\text {the }, n} /\left[\gamma\left(A_{e} a_{n}-\gamma_{\text {the }, n} D_{e} \sum_{i=1}^{n-1} a_{i}-\gamma_{\text {the }, n} A_{e} \sum_{i=n+1}^{N} a_{i}-\gamma_{\text {the }, n} B_{e}\right)\right]$.

Similarly, the following theorem explores the security performance in term of IP over Nakagami- $m$ fading channels with IQI.

Theorem 2. The exact closed-form expressions for the IP of $D_{n}$ can be expressed as

- Non-ideal conditions

$$
P_{\text {int }}^{n, n i}=1-\left[1-\sum_{g_{i}=0}^{\alpha_{i}-1} \frac{e^{-\frac{\delta_{n}}{\beta_{i}}}}{g_{i} !}\left(\frac{\delta_{n}}{\beta_{i}}\right)^{g_{i}}\right]^{K},
$$

for $0<\gamma_{\text {the }, n}<\frac{A_{e} a_{n}}{D_{e} \sum_{i=1}^{n-1} a_{i}+A_{e} \sum_{i=n+1}^{N} a_{i}+B_{e}}$, and $P_{\mathrm{int}}^{n, n i}=0$ for $0<\gamma_{\text {the }, n}>\frac{A_{e} a_{n}}{D_{e} \sum_{i=1}^{n-1} a_{i}+A_{e} \sum_{i=n+1}^{N} a_{i}+B_{e}}$.

- $\quad$ Ideal conditions $\left(\varsigma_{t}=\varsigma_{r_{j}}=1.0, \phi_{t}=\phi_{r_{j}}=0^{\circ}\right)$

$$
P_{\mathrm{int}}^{n, i d}=1-\left[1-\sum_{g_{i}=0}^{\alpha_{i}-1} \frac{e^{-\frac{\delta}{\beta_{i}}}}{g_{i} !}\left(\frac{\delta}{\beta_{i}}\right)^{g_{i}}\right]^{K},
$$

for $0<\gamma_{\text {the }, n}<\frac{a_{n}}{\sum_{i=n+1}^{N} a_{i}}$, and $P_{\mathrm{int}}^{n, i d}=0$ for $\gamma_{\text {the }, n}>\frac{a_{n}}{\sum_{i=n+1}^{N} a_{i}}$.

where $\delta=\gamma_{\text {the }, n} /\left[\gamma\left(a_{n}-\gamma_{\text {the }, n} \sum_{i=n+1}^{N} a_{i}\right)\right]$.

Proof. See Appendix B.

Remark 1. Theorems 1 and 2 have provided some insights that for the reliability and security of the considered system, there exist upper bounds for the effective SNR, $A_{n} a_{p} /\left(D_{p} \sum_{i=1}^{p-1} a_{i}+A_{n} \sum_{i=p+1}^{N} a_{i}+B_{n}\right)$, and $A_{e} a_{n}-\gamma_{\text {the }, n} D_{e} \sum_{i=1}^{n-1} a_{i}-\gamma_{\text {the }, n} A_{e} \sum_{i=n+1}^{N} a_{i}-\gamma_{\text {the },} B_{e}$, otherwise, the outage event always occurs and the eavesdrop event can not occurs. Moreover, IQI parameters has detrimental effects on the OP of the considered system, while it is always beneficial to the IP of the considered system.

\subsection{Asymptotic Analysis}

To gain more insights, we will carry out the asymptotic analysis for the OP of the considered system over Nakagami- $m$ fading channels in the presence of IQI in the high SNR region. According 
to [47], the CDF of the channel gain $\rho_{i}$ of the unordered and ordered channels can be respectively approximated as

$$
\begin{gathered}
F_{\rho_{i}}^{\infty}(x) \approx \frac{x^{\alpha_{i}}}{\alpha_{i} ! \beta_{i}{ }^{\alpha_{i}}}, \\
F_{\rho_{i}}^{\infty}(x)=\frac{N !}{(N-n) ! n !}\left(\frac{1}{\alpha_{i} !}\right)^{n}\left(\frac{x}{\beta_{i}}\right)^{\alpha_{i} n} .
\end{gathered}
$$

Corollary 1. At high SNRs, the asymptotic outage probability for $D_{n}$ can be expressed as

- $\quad$ Non-ideal conditions

$$
P_{o u t, \infty}^{D_{n}, n i}=\frac{N !}{(N-n) ! n !}\left(\frac{1}{\alpha_{i} !}\right)^{n M}\left(\frac{\theta_{n}^{*}}{\beta_{i}}\right)^{\alpha_{i} n M} .
$$

- Ideal conditions $\left(\varsigma_{t}=\varsigma_{r_{j}}=1.0, \phi_{t}=\phi_{r_{j}}=0^{\circ}\right)$

$$
P_{o u t, \infty}^{D_{n, n} n}=\frac{N !}{(N-n) ! n !}\left(\frac{1}{\alpha_{i} !}\right)^{n M}\left(\frac{\theta}{\beta_{i}}\right)^{\alpha_{i} n M} .
$$

\subsection{Diversity Order}

In this section, we focus on the diversity order, and the definition of the diversity order is given by [48]

$$
d_{n}=-\lim _{\gamma \rightarrow \infty} \frac{\log \left(P_{\text {out }}^{D_{n}, \infty}\right)}{\log \gamma} .
$$

Based on the derived asymptotic results, the diversity order for the OP of the considered system are provided in the following corollary.

Corollary 2. The diversity order of the $n$-th user is expressed as

$$
d_{n}=\alpha_{i} n M
$$

Remark 2. Corollary 2 reveals that diversity order are only rely on the shape parameter $\alpha$, user order $n$ and the number of antennas of destination node. In addition, the diversity order for ideal conditions and non-ideal conditions are the same. This means that IQI parameters are irrelative to the diversity order of the considered system.

\section{Numeric Results}

In this section, the correctness of theoretical analysis is verified by Monte Carlo simulations. We obtain the Monte Carlo simulation results with $10^{7}$ iterations. Unless other stated, the simulated parameter values are set as in Table 1.

Table 1. Table of Parameters for numerical results.

\begin{tabular}{ll}
\hline Monte Carlo Simulations Repeated & $\mathbf{1 0}^{\mathbf{7}}$ Iterations \\
\hline The number of legitimate destinations & $N=3$ \\
The number of antennas & $M=K=2$ \\
Power sharing coefficients of NOMA & $a_{1}=1 / 2, a_{2}=1 / 3, a_{3}=1 / 6$ \\
Noise power & $N_{0}=1$ \\
Targeted data rates (OP) & $\gamma_{t h 1}=0.9, \gamma_{t h 2}=1.5, \gamma_{t h 3}=3$ \\
Targeted data rates (IP) & $\gamma_{t h e, 1}=0.87, \gamma_{t h e, 2}=1.5$, and $\gamma_{t h e, 3}=3.6$ \\
The fading parameters of the channel & $\alpha_{1}=\alpha_{2}=\alpha_{3}=\alpha=1, \beta_{1}=\beta_{2}=\beta_{3}=0.5$ \\
Ideal RF front end & $\varsigma_{t}=\varsigma_{r_{j}}=1.0, \phi_{t}=\phi_{r_{j}}=0^{\circ}$ \\
IQI parameters & $\varsigma_{t}=\varsigma_{r_{j}}=1.14, \phi_{t}=\phi_{r_{j}}=7^{\circ}$ \\
\hline
\end{tabular}


Figure 2 shows the OP and IP versus the average SNR for different NOMA users. For the purpose of comparison, the OP and IP curves in the presence of ideal conditions are provided. We can see that the outage performance of each user under ideal conditions outperforms that of the IQI conditions; and the IP under ideal conditions is greater than that under IQI conditions. These results are consistent with the conclusion of Remark 1. In addition, we can conclude that IQI parameter has more severe impact on the OP and IP of $D_{2}$ and $D_{3}$ than that of $D_{1}$. This happens because during SIC operation, the effects of IQI on $D_{2}$ and $D_{3}$ can not be fully eliminated, and there are still some residual interferences from $D_{1}$. On the other hand, these residual interferences are always beneficial to the IP performance. Finally, we can also see that there exists a trade-off between reliability and security.

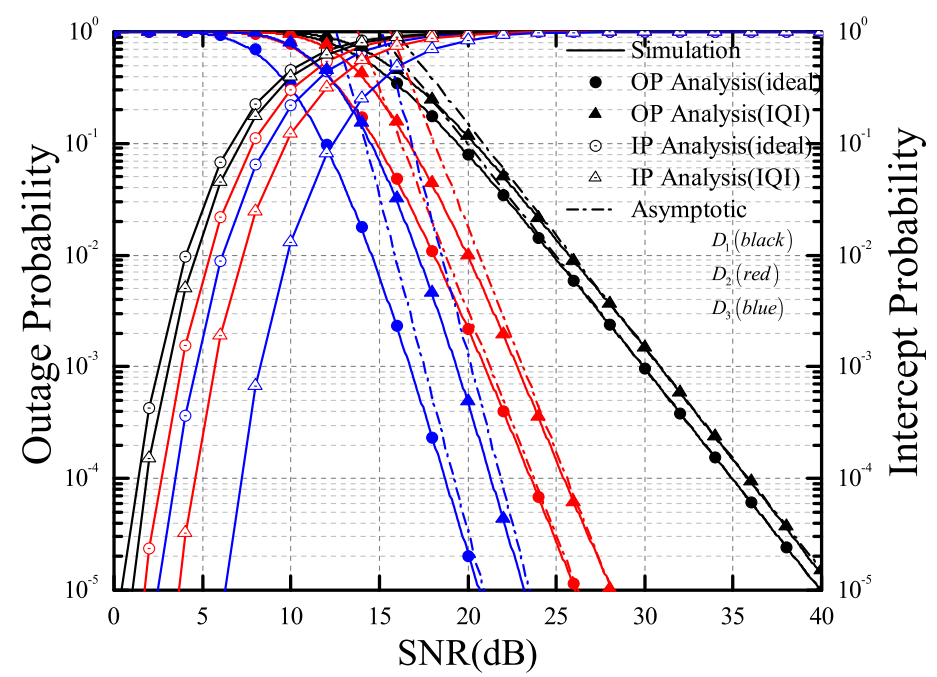

Figure 2. Outage probability (OP) and intercept probability (IP) versus the average signal-to-noise ratio (SNR) for different NOMA users.

Figure 3 plots the OP and IP versus $M$ and $K$ for different users, respectively. In this simulation, we assume $S N R=15 \mathrm{~dB}$ for OP and $S N R=10 \mathrm{~dB}$ for IP. From Figure 3, we can observe that the change in the number of antennas has the same effects on the OP and IP of each user. Specifically, we can observe that the OP decreases as the number of antennas of destination node $(M)$ increasing, while IP increases as the number of antennas of eavesdropper node $(K)$ increasing. This means that the increase of $M$ can improve the reliability of the receiver, and the increase of $K$ can reduce the security performance. Moreover, we can observe that there are optimal values to ensure the balance between OPs and IPs.

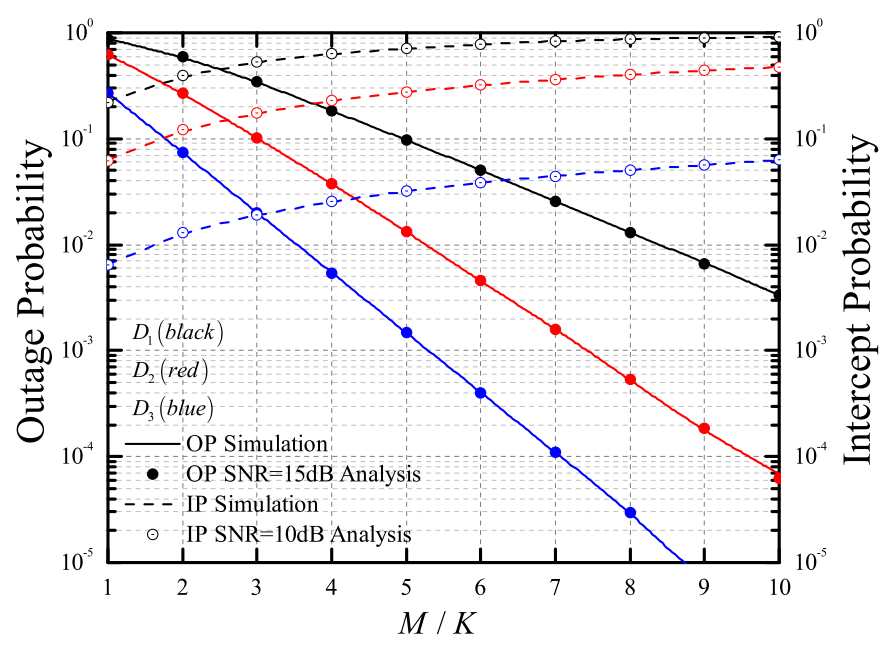

Figure 3. OP and IP versus $M / K$ for different NOMA users. 
Figure 4 plots the OP versus the power allocation coefficient $a_{1}$ for the first user of a three-user NOMA system. In this simulation, we set $S N R=13 \mathrm{~dB}$ and $a_{2}=2\left(1-a_{1}\right) / 3, a_{3}=\left(1-a_{1}\right) / 3$, $\gamma_{t h 1}=0.9, \gamma_{t h 2}=0.9, \gamma_{t h 3}=1.2$. For comparison, the curves for the OPs of ideal conditions and IQI conditions are provided. We can observe that IQI can cause a significant increase in the OP of the system, which depends on the power allocation coefficient of the NOMA users. When the power allocation coefficient $a_{1}$ is larger than 0.8 , outage events will always happen. This is because for large $a_{1}$, there is no power to allocate to $a_{2}$ and $a_{3}$. In addition, we can also observe that the increase of the number of user antennas will lead to a sharp drop in the OP, it also mainly depends on the power allocation parameters. At the same time, there exists an optimal $a_{1}$ to minimize OP, i.e., the outage performance is the best when $a_{1} \approx 0.54$.

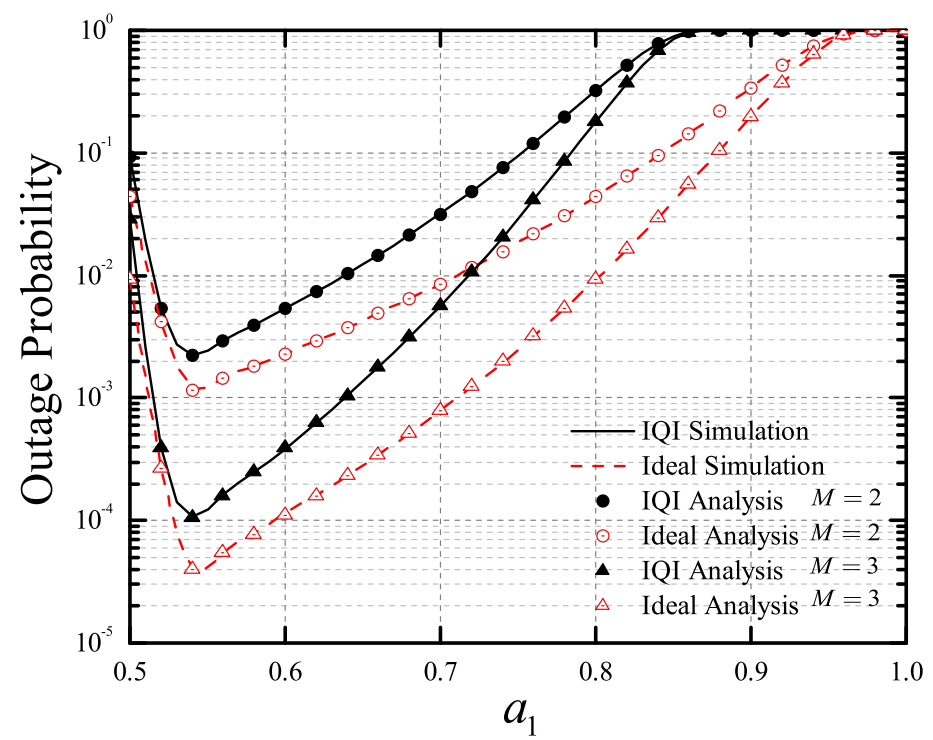

Figure 4. OP versus $a_{1}$ for different numbers of antennas.

Figure 5 shows the IP of a three-user NOMA system versus the power allocation coefficient $a_{1}$ in the presence of IQI. In this simulation, we set $a_{2}=2\left(1-a_{1}\right) / 3, a_{3}=\left(1-a_{1}\right) / 3, S N R=6 \mathrm{~dB}$, $\gamma_{\text {the } 1}=0.9, \gamma_{\text {the } 2}=1.4, \gamma_{\text {the }}=2.5$. From Figure 5 , we can obtain that IQI has slight effects on the IP of $D_{1}$, especially for large value of $a_{1}$, while other users experience a significant drop in IP due to IQI and few power allocation. It can be clearly seen from Figure 5 that as the power distribution coefficient $a_{1}$ increases, the IP of the $D_{1}$ also becomes larger, and the IP of the $D_{2}$ and the $D_{3}$ become smaller. This is because when $a_{1}$ increases, the power allocated by the $D_{1}$ becomes large, thus causing the security thereof to be lowered.

Figure $6 \mathrm{a}, \mathrm{b}$ show the OP and IP of a three-user NOMA system versus IQI amplitude mismatch parameters $\varsigma_{t}=\varsigma_{r}$ for different target rates. For the convenience of comparison, we set $\phi_{t}=\phi_{r}=0^{\circ}$. In this simulation, we set $\left\{\gamma_{t h 1}, \gamma_{t h 2}, \gamma_{t h 3}\right\}=\{0.9,1.5,3\}$ and $\left\{\gamma_{\text {the } 1}, \gamma_{\text {the } 2}, \gamma_{\text {thes }}\right\}=\{0.87,1.5,3.6\}$ for Figure $6 \mathrm{a},\left\{\gamma_{\text {th } 1}, \gamma_{\text {th } 2}, \gamma_{\text {th } 3}\right\}=\{0.7,1.7,1.7\}$ and $\left\{\gamma_{\text {the } 1}, \gamma_{\text {the } 2}, \gamma_{\text {the }}\right\}=\{0.87,1.5,1.5\}$ for Figure $6 \mathbf{b}$. Figure 6a shows that the outage performance can be reduced as $\varsigma_{t}$ increases, especially for $D_{2}$ and $D_{3}$. At the same time, the IPs for three users decrease as $\varsigma_{t}$ increases, which is more obvious for $D_{2}$ and $D_{3}$. This means that although amplitude mismatch parameters can reduce the reliability, it can enhance the security performance of the system. For Figure $6 b$, there is a different phenomenon for the OP that $D_{2}$ and $D_{3}$ suffer more effects from IQI due to different rate threshold. The similar phenomenon also happens in IP. By comparing the Figure $6 \mathrm{a}, \mathrm{b}$, we can observe that $D_{1}$ is quite robust to the variation of amplitude parameters of IQI in these two cases, even at a higher IQI level. For the moderate or high level of IQI amplitude parameters, the OPs of the other two users increase significantly, while the IPs decrease. In addition, we can also observe that the order of OP and IP between users is changed due to the change of target rate of each user, which is due to the power domain multiplexing of NOMA, 
which allocates less power to high-order users, which makes them more sensitive to noise, interference and impairments.

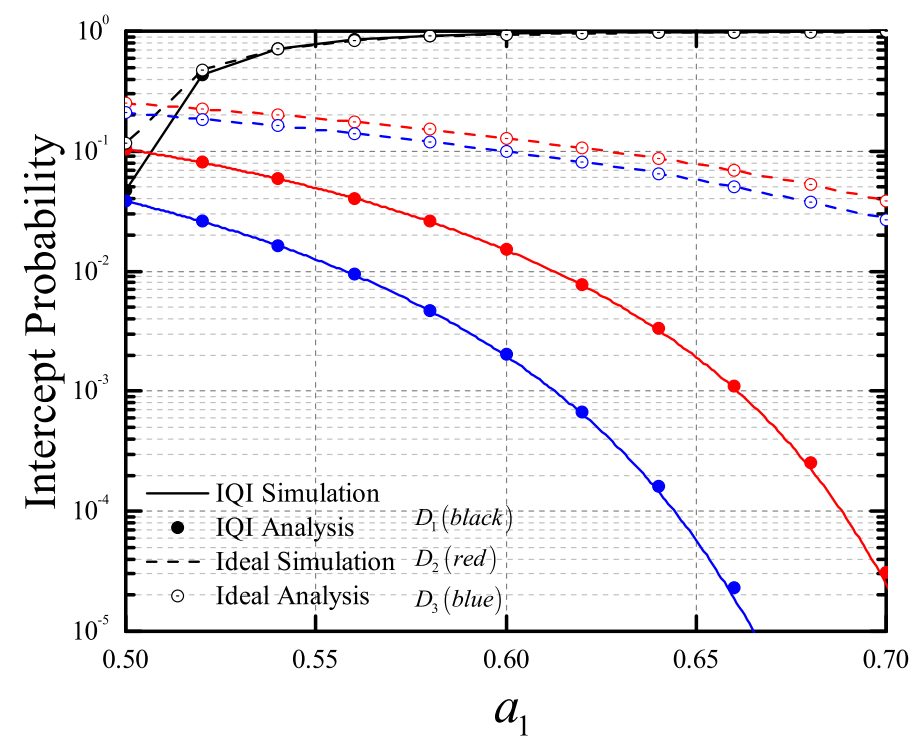

Figure 5. IP versus $a_{1}$ for different NOMA users.

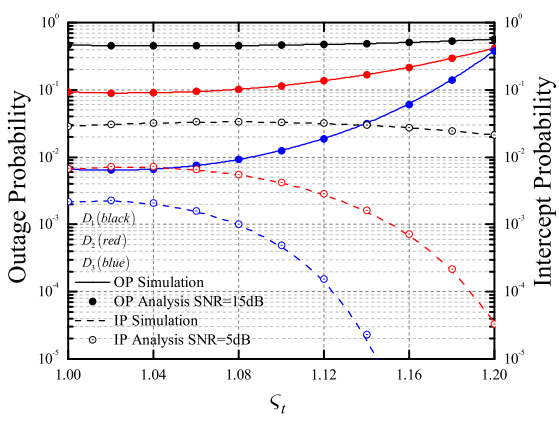

(a)

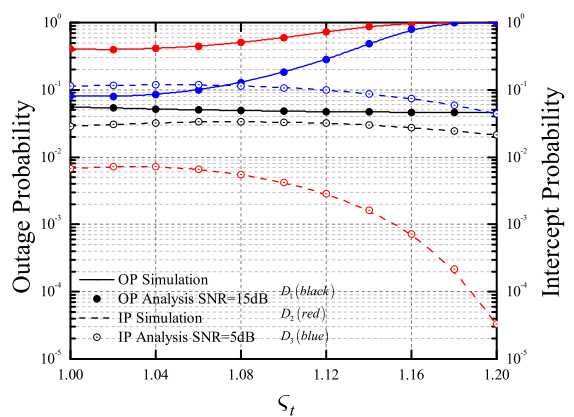

(b)

Figure 6. OP and IP versus amplitude mismatch $\varsigma_{t}\left(\varsigma_{t}=\varsigma_{r}\right)$ for different target rate (a): $\left\{\gamma_{t h 1}, \gamma_{t h 2}, \gamma_{t h 3}\right\}=\{0.9,1.5,3\} ;\left\{\gamma_{t h e}, \gamma_{t h e 2}, \gamma_{t h e 3}\right\}=\{0.87,1.5,3.6\} . \quad(\mathbf{b}):\left\{\gamma_{t h 1}, \gamma_{t h 2}, \gamma_{t h 3}\right\}=$ $\{0.7,1.7,1.7\} ;\left\{\gamma_{\text {the } 1}, \gamma_{\text {the } 2}, \gamma_{\text {the }}\right\}=\{0.87,1.5,1.5\}$.

Figure 7a,b show the OP and IP of a three-user NOMA versus the phase mismatch for different target rates. For this consideration, we set $\varsigma_{t}=\varsigma_{r}=1, \phi_{t}=\phi_{r}$. It can be seen that the phase parameters of IQI have smaller effects on $D_{1}$ for the two cases, and it has a greater impact on $D_{2}$ and $D_{3}$. The reason for this is that $D_{1}$ has poor channel conditions. With the increase of phase, the OP of each user increases and the IP decreases. We can also observe that the outage event occurs when the phases are $\phi_{t}=\phi_{r} \geq 13^{\circ}$. This happens because that when $\gamma_{t h j}>A a_{j} /\left(D \sum_{i=1}^{j-1} a_{i}+A \sum_{i=j+1}^{N} a_{i}+B\right)$, the OP is always 1 . Finally, by comparing Figure 6 with Figure 7, we clearly observe that the amplitude and phase have the same effects on the OP and IP.

Figure 8 plots the OP and the asymptotic results versus average transmit SNR for different fading parameters $\alpha$ and antenna number $M$. In this simulation, we set $\gamma_{t h 1}=0.8, \gamma_{t h 2}=1.5, \gamma_{t h 3}=2$. We can observe from the Figure 8 that for each user, when antenna number is the same, the corresponding OP becomes smaller with the increase of fading parameters. When the fading parameters $\alpha$ have the same values, the OP decreases with the increase of antenna number $M$. It is proved that the reliability of the system will be improved with the increase of channel fading parameters or the number of 
antenna at the receiver. In addition, we can also see from the Figure 8 that the asymptotic curves of $D_{1}(M=2, \alpha=1)$ and $D_{2}(M=1, \alpha=1)$ is parallel, and the asymptotic curves of $D_{1}(M=2, \alpha=2)$ and $D_{2}(M=2, \alpha=1)$ are also parallel, which verifies the diversity order of derived in (33), and their corresponding diversity order is the same.

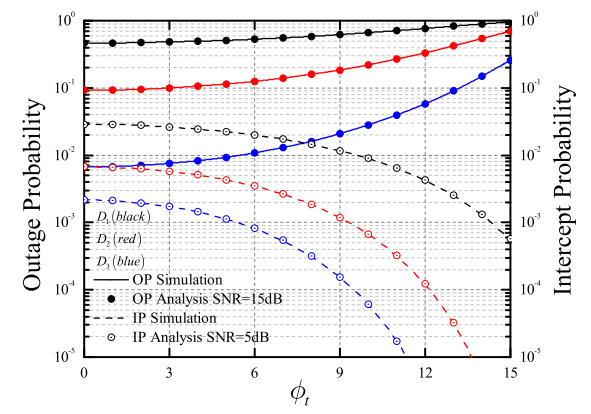

(a)

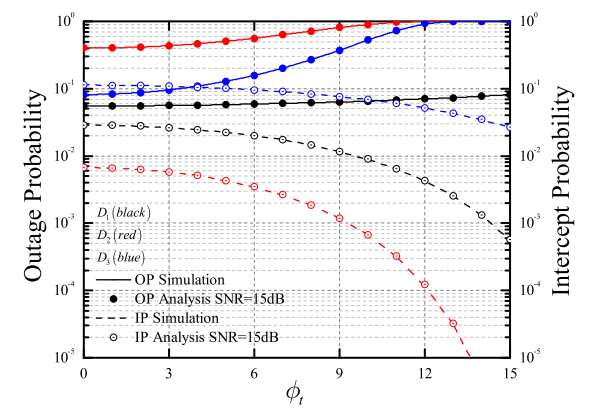

(b)

Figure 7. OP and IP versus phase mismatch $\phi_{t}\left(\phi_{t}=\phi_{r}\right)$ for different target rate $(\mathbf{a}):\left\{\gamma_{t h 1}, \gamma_{t h 2}, \gamma_{t h 3}\right\}=$ $\{0.9,1.5,3\} ; \quad\left\{\gamma_{\text {the } 1}, \gamma_{\text {the } 2}, \gamma_{\text {the }}\right\}=\{0.87,1.5,3.6\} . \quad(\mathbf{b}): \quad\left\{\gamma_{\text {th } 1}, \gamma_{\text {th } 2}, \gamma_{\text {th } 3}\right\}=\{0.7,1.7,1.7\}$; $\left\{\gamma_{\text {the } 1}, \gamma_{\text {the } 2}, \gamma_{\text {the }}\right\}=\{0.87,1.5,1.5\}$

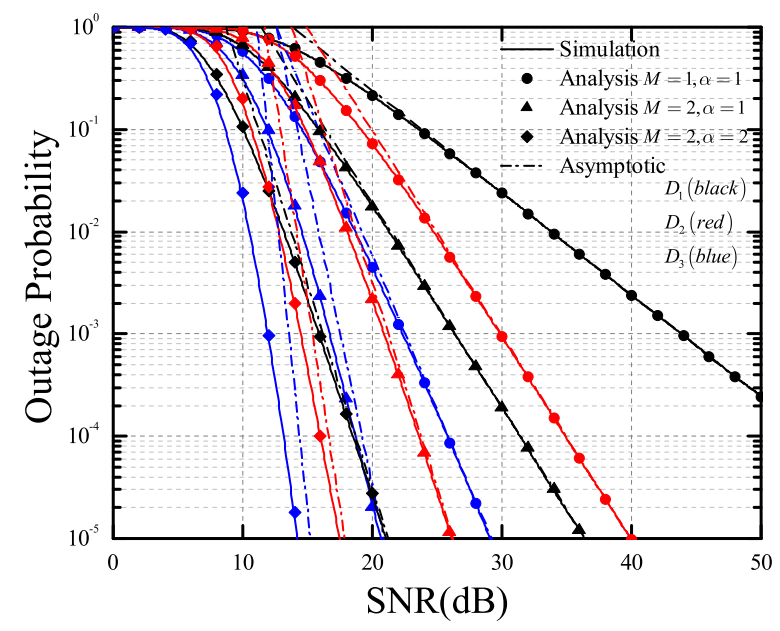

Figure 8. OP versus SNR for different $\alpha$ and $M$.

\section{Conclusions}

In this paper, we investigate the effects of IQI on SIMO-NOMA systems over Nakagami- $m$ fading channel. Considering the practical factor of IQI at TX and RX, the closed-form analytical expressions of IP and OP of the considered system under IQI are derived. Also, the diversity order of the legitimate users has been discussed in detail, and the results of the analysis are verified extensively by Monte-Carlo simulation. These results have shown that both the amplitude and phase parameters of the IQI reduce the reliability of the users and improve the security. The severity of the performance degradation depends on several factors, including the power allocation ratio, the transmission SNR ratio, the number of antennas, and the order of considered users. In addition, IQI can affect the optimal power allocation coefficient among users.

In future work, cooperative communications can be combined with our considered system to further improve reliability and security performance. In addition, the Nakagami- $m$ channel model can also be applied to other scenarios, such as the cloud environment composed of vehicles [49], which constitutes our future research work. 
Author Contributions: Conceptualization, X.L.; data curation; X.L. and M.Z.; methodology, X.L.; software, M.Z.; supervision, X.L., C.Z., W.U.K., J.W., K.M.R. and R.K.; validation, X.L., M.Z., C.Z., W.U.K., J.W., K.M.R. and R.K.; writing-original draft preparation, X.L., M.Z.; writing-review and editing, M.Z.

Funding: This work was supported in part by the Henan Scientific and Technological Research Project under Grant 182102210307, in part by the Doctoral Scientific Funds of Henan Polytechnic University under Grant B2017-55, in part by the Fundamental Research Funds for the Universities of Henan Province under Grant NSFRF180309, in part by the Outstanding Youth Science Foundation of Henan Polytechnic University under Grant J2019-4, in part by the Key Scientific Research Projects of Higher Education Institutions in Henan Province Grant 20A510007, and in part by the National Natural Science Foundation of China Grant 61601414 and 61801435).

Conflicts of Interest: The authors declare no conflict of interest.

\section{Appendix A. Proof of Theorem 1}

Based on the fact that the $D_{n}$ needs to decode all of the user's information, whose the channel conditions of their gains are worse than its own, and using (13), the outage probability of $D_{n}$ in (19) can be expressed as

$$
\begin{aligned}
P_{\text {out }}^{D_{n}} & =1-\operatorname{Pr}\left(E_{n, 1}^{S D} \cap \cdots E_{n, n}^{S D}\right) \\
& =1-\operatorname{Pr}\left(\rho_{\rho_{S D_{n, \widehat{m}}}}>\max _{1 \leq p \leq n} \theta_{p}\right) \\
& =F_{\rho_{S D_{n, \hat{m}}}}\left(\widehat{\theta}_{n}\right) \\
& =\frac{N !}{(n-1) !(N-n) !} \sum_{l=0}^{N-n}\left(\begin{array}{l}
N-n \\
l
\end{array}\right) \frac{(-1)^{l}}{n+l} \sum_{b=0}^{M(n+l)} U_{b}(-1)^{b} U_{b} V_{b}\left(\widehat{\theta}_{n}\right)^{\bar{b}} e^{-\frac{\widehat{\theta}_{n}}{\beta_{i}} b}
\end{aligned}
$$

where $\widehat{\theta}_{n}=\max _{1 \leq p \leq n} \theta_{p}$, otherwise, using the CDF of the channel gain $\rho_{s d_{n, m}}$ in (4) and through some simple mathematical calculations, the $\mathrm{CDF}$ of the channel gain $\rho_{S D_{n, \hat{m}}}$ can be obtained as

$$
\begin{aligned}
F_{\rho_{S D_{n, \hat{m}}}}(x) & =\operatorname{Pr}\left(\rho_{\rho_{S D_{n, \hat{m}}}}<x\right) \\
& =\frac{N !}{(n-1) !(N-n) !} \sum_{l=0}^{N-n}\left(\begin{array}{l}
N-n \\
k
\end{array}\right) \frac{(-1)^{l}}{n+l}\left[1-\sum_{g_{i}=0}^{\alpha_{s d . n}-1} \frac{e^{-\frac{x}{\beta_{s d, n}}}}{g_{i} !}\left(\frac{x}{\beta_{s d, n}}\right)^{g_{i}}\right]^{M(n+l)}
\end{aligned}
$$

Substituting (A2) into (A1), (21) can be obtained, the proof is completed. In the special case of ideal conditions, the outage probability expression can be obtained by setting $\varsigma_{t}=\varsigma_{r_{j}}=1.0, \phi_{t}=\phi_{r_{j}}=0^{\circ}$ in Theorem 1.

\section{Appendix B. Proof of Theorem 2}

Substituting (18) into (25), the Intercept probability can be expressed as

$$
\begin{aligned}
& P_{\text {int }}^{n}=\operatorname{Pr}\left(\gamma_{S E}^{n}>\gamma_{\text {then }}\right) \\
& =\operatorname{Pr}\left(\frac{\rho_{E_{\widehat{k}}} \gamma A_{e} a_{k}}{\rho_{E_{\widehat{k}}} \gamma D_{e} \sum_{i=1}^{k-1} a_{i}+\rho_{E_{\widehat{k}}} \gamma A_{e} \sum_{i=k+1}^{N} a_{i}+\rho_{E_{\widehat{k}}} \gamma B_{e}+C_{e}}>\gamma_{\text {then }}\right) \\
& =1-F_{\rho_{E_{\widehat{k}}}}\left(\delta_{n}\right)
\end{aligned}
$$

In what follows, $P_{\text {int }}^{n}$ will be addressed. According to the CDF of the the channel gain $\rho_{E}$ in (2), the $\mathrm{CDF}$ of the channel gain $\rho_{E_{\widehat{k}}}$ can be calculated as: 


$$
\begin{aligned}
F_{\rho_{E_{\widehat{k}}}}(x) & =\operatorname{Pr}\left(\rho_{E_{\widehat{k}}}<x\right) \\
& =\left[F_{\rho_{E}}(x)\right]^{K} \\
& =\left[1-\sum_{g_{i}=0}^{\alpha_{i}-1} \frac{e^{-\frac{x}{\beta_{i}}}}{g_{i} !}\left(\frac{x}{\beta_{i}}\right)^{g_{i}}\right]^{K}
\end{aligned}
$$

Substituting (A4) into (A3), we can obtain the (26), the proof is completed. In the special case of ideal conditions, the outage probability expression can be obtained by setting $\varsigma_{t}=\varsigma_{r_{j}}=1.0$, $\phi_{t}=\phi_{r_{j}}=0^{\circ}$ in Theorem 2 .

\section{References}

1. Li, X.; Li, J.; Mathiopoulos, P.T.; Zhang, D. Joint Impact of Hardware Impairments and Imperfect CSI on Cooperative SWIPT NOMA Multi-Relaying Systems. In Proceedings of the 2018 IEEE/CIC International Conference on Communications in China (ICCC), Beijing, China, 16-18 August 2018; pp. 95-99.

2. Zeng, J.; Lv, T.; Liu, R.P. Investigation on Evolving Single-Carrier NOMA Into Multi-Carrier NOMA in 5G. IEEE Access 2018, 6, 48268-48288. [CrossRef]

3. Li, X.; Liu, M.; Deng, C.; Mathiopoulos, P.T.; Ding, Z.; Liu, Y. Full-Duplex Cooperative NOMA Relaying Systems with I/Q Imbalance and Imperfect SIC. IEEE Wirel. Commun. Lett. 2019. [CrossRef]

4. Li, X.; Li, J.; Liu, Y.; Ding, Z.; Nallanathan, A. Residual Transceiver Hardware Impairments on Cooperative NOMA Networks. IEEE Trans. Wirel. Commun. 2019. [CrossRef]

5. Khan, W.U.; Jameel, F.; Ristaniemi, T.; Khan, S.; Sidhu, G.A.S.; Liu, J. Joint Spectral and Energy Efficiency Optimization for Downlink NOMA Networks. IEEE Trans. Cogn. Commun. Netw. 2019. [CrossRef]

6. Niharika, G. Performance comparison of modulation schemes for Downlink NOMA. In Proceedings of the 2018 2nd International Conference Inventive System Control, Coimbatore, India , 19-20 January 2018; pp. 1431-1433.

7. Abbasi, O.; Ebrahimi, A.; Mokari, N. NOMA Inspired Cooperative Relaying System Using an AF Relay. IEEE Wirel. Commun. Lett. 2019, 8, 261-264. [CrossRef]

8. Yue, X.; Liu, Y.; Kang, S.; Nallanathan, A. Performance Analysis of NOMA with Fixed Gain Relaying Over Nakagami- $m$ Fading Channels. IEEE Access 2017, 5, 5445-5454. [CrossRef]

9. Men, J.; Ge, J. Performance analysis of non-orthogonal multiple access in downlink cooperative network. IET Commun. 2015, 9, 2267-2273. [CrossRef]

10. Ding, Z.; Fan, P.; Poor, H.V. Random beamforming in millimeterwave NOMA networks. IEEE Access 2017, 5, 7667-7681. [CrossRef]

11. Arzykulov, S.; Nauryzbayev, G.; Tsiftsis, T.A.; Maham, B.; Abdallah, M. On the Outage of Underlay CR-NOMA Networks with Detect-and-Forward Relaying. IEEE Trans. Cogn. Commun. Netw. 2019, 5, 795-804. [CrossRef]

12. Mirabbasi, S.; Martin, K. Classical and modern receiver architectures. IEEE Commun. Mag. 2000, 38, 132-139. [CrossRef]

13. Li, J.; Matthaiou, M.; Svensson, T. I/Q Imbalance in AF Dual-Hop Relaying: Performance Analysis in Nakagami- $m$ Fading. IEEE Trans. Commun. 2014, 62, 836-847. [CrossRef]

14. Chen, L.; Helmy, A.G.; Yue, G.; Li, S.; Al-Dhahir, N. Performance Analysis and Compensation of Joint TX/RX I/Q Imbalance in Differential STBC-OFDM. IEEE Trans. Veh. Technol. 2017, 66, 6184-6200. [CrossRef]

15. Hamila, R.; Özdemir, Ö.; Al-Dhahir, N. Beamforming OFDM Performance Under Joint Phase Noise and I/Q Imbalance. IEEE Trans. Veh. Technol. 2016, 65, 2978-2989. [CrossRef]

16. Faruk, M.S.; Kikuchi, K. Compensation for In-Phase/Quadrature Imbalance in Coherent-Receiver Front End for Optical Quadrature Amplitude Modulation. IEEE Photon. J. 2013, 5, 7800110. [CrossRef]

17. Ruihao, C.; Xiuli, Y.; Yuyao, S.; Yongqing, W. Method for the compensation of transmitter I/Q imbalance based on the pre-distortion of baseband signals. In Proceedings of the 2017 9th International Conference Advanced Infocomm Technology, Chengdu, China, 22-24 November 2017; pp. 313-317. 
18. Nam, W.; Roh, H.; Lee, J.; Kang, I. Blind Adaptive I/Q Imbalance Compensation Algorithms for Direct-Conversion Receivers. IEEE Signal Process. Lett. 2012, 19, 475-478. [CrossRef]

19. Selim, B.; Muhaidat, S.; Sofotasios, P.; Sharif, B.; Stouraitis, T.; Karagiannidis, G.; Al-Dhahir, N. Performance Analysis of Non-Orthogonal Multiple Access Under I/Q Imbalance. IEEE Access 2018, 6, 18453-18468. [CrossRef]

20. Khan, W.U. Maximizing physical layer security in relay-assisted multicarrier nonorthogonal multiple access transmission. Internet Technol. Lett. 2019, 2. [CrossRef]

21. Chen, X.; Guo, L.; Li, X.; Dong, C.; Lin, J. Secrecy rate optimization for cooperative cognitive radio networks aided by a wireless energy harvesting jammer. IEEE Access 2018, 6, 34127-34134. [CrossRef]

22. Yang, N.; Wang, L.; Geraci, G.; Elkashlan, M.; Yuan, J.; di Renzo, M. Safeguarding 5G wireless communication networks using physical layer security. IEEE Commun. Mag. 2015, 53, 20-27. [CrossRef]

23. Bloch, M.; Barros, J.; Rodrigues, M.R.D.; McLaughlin, S.W. Wireless information-theoretic security. IEEE Trans. Inf. Theory 2008, 54, 2515-2534. [CrossRef]

24. Liang, L.; Wei, D.; Li, M. Wireless Physical Layer Security Based on Channel Pre-Compensation for PolSK Modulated Systems. In Proceedings of the 2017 IEEE International Conference Communication Software and Networks, Guangzhou, China, 6-8 May 2017; pp. 357-361.

25. Wang, H.; Luo, M.; Yin, Q.; Xia, X. Hybrid Cooperative Beamforming and Jamming for Physical-Layer Security of Two-Way Relay Networks. IEEE Trans. Inf. Forensics Secur. 2013, 8, 2007-2020. [CrossRef]

26. Jia, S.; Zhang, J.; Zhao, H.; Xu, Y. Performance analysis of physical layer security over $\alpha-\eta-\kappa-\mu$ fading channels. China Commun. 2018, 15, 138-148. [CrossRef]

27. Ammari, M.; Fortier, P. Physical layer security of multiple-input-multiple-output systems with transmit beamforming in Rayleigh fading. IET Commun. 2015, 9, 1096-1103. [CrossRef]

28. Abolpour, M.; Mirmohseni, M.; Aref, M. Outage Performance in Secure Cooperative NOMA. In Proceedings of the 2019 Iran Workshop on Communication and Information Theory (IWCIT), Tehran, Iran, 24-25 April 2019; pp. 1-6.

29. Shim, K.; Do, T.; An, B. Improving Physical Layer Security of NOMA Networks by Using Opportunistic Scheduling. In Proceedings of the 2018 Tenth International Conference Ubiquitous and Future Networks (ICUFN), Prague, Czech Republic, 3-6 July 2018; pp. 262-267.

30. Satrya, G.B.; Shin, S.Y. Security enhancement to successive interference cancellation algorithm for non-orthogonal multiple access (NOMA). In Proceedings of the 2017 IEEE 28th Annual International Symposium Pers., Indoor, Mobile Radio Communications, Montreal, QC, Canada, 8-13 October 2017; pp. 1-5.

31. Zheng, B.; Wen, M.; Chen, F.; Tang, J.; Ji, F. Secure NOMA Based Full-Duplex Two-Way Relay Networks with Artificial Noise against Eavesdropping. In Proceedings of the 2018 IEEE International Conference Communications, Kansas City, MO, USA, 20-24 May 2018; pp. 1-6.

32. Lei, H.; Zhang, J.; Park, K.; Xu, P.; Zhang, Z.; Pan, G. Secrecy Outage of Max-Min TAS Scheme in MIMO-NOMA Systems. IEEE Trans. Veh. Technol. 2018, 67, 6981-6990. [CrossRef]

33. Zaghdoud, N.; Alouane, W.; Boujemaa, H.; Touati, F. Secure Performance of AF and DF Relaying in Cooperative Noma Systems. In Proceedings of the 2019 19th International Conference Sciences and Techniques of Automatic Control and Computer Engineering (STA), Sousse, Tunisia, 24-26 March 2019; pp. 614-619.

34. Liu, Y.; Xing, H.; Pan, C.; Nallanathan, A.; Elkashlan, M.; Hanzo, L. Multiple-Antenna-Assisted Non-Orthogonal Multiple Access. IEEE Wirel. Commun. 2018, 25, 17-23. [CrossRef]

35. Lei, H.; Zhang, J.; Park, K.; Xu, P.; Ansari, I.S.; Pan, G.; Alomair, B.; Alouini, M.S. On Secure NOMA Systems WITH Transmit Antenna Selection Schemes. IEEE Access 2017, 5, 17450-17464. [CrossRef]

36. Gomez, G.; Martin-Vega, F.; Javier, F.; Liu, Y.; Elkashlan, M. Physical Layer Security in Uplink NOMA Multi-Antenna Systems with Randomly Distributed Eavesdroppers. IEEE Access 2019, 7, 70422-70435. [CrossRef]

37. Li, X.; Huang, M.; Zhang, C.; Deng, D.; Rabie, K.; Ding, Y.; Du, J. Security and Reliability Performance Analysis of Cooperative Multi-Relay Systems with Nonlinear Energy Harvesters and Hardware Impairments. IEEE Access 2019, 7, 102644-102661. [CrossRef]

38. Korn, I.; Fonseka, J.P. FSK with Limiter-Discriminator Detector in Nakagami Fading Channels with and without Selection Combining. IEEE Trans. Veh. Technol. 2006, 55, 1934-1940. [CrossRef] 
39. Lei, H.; Ansari, I.S.; Pan, G.; Alomair, B.; Alouini, M.S. Secrecy Capacity Analysis Over $\alpha-\mu$ Fading Channels. IEEE Commun. Lett. 2017, 21, 1445-1448. [CrossRef]

40. Sun, J.; Li, X.; Huang, M.; Ding, Y.; Jin, J.; Pan, G. Performance analysis of physical layer security over $\kappa-\mu$ shadowed fading channels. IET Commun. 2018, 12, 970-975. [CrossRef]

41. Bjornson, E.; Matthaiou, M.; Debbah, M. A New Look at Dual-Hop Relaying: Performance Limits with Hardware Impairments. IEEE Trans. Commun. 2013, 61, 4512-4525. [CrossRef]

42. Li, X.; Li, J.; Li, L. Performance Analysis of Impaired SWIPT NOMA Relaying Networks Over Imperfect Weibull Channels. IEEE Syst. J. 2019, 1-4. [CrossRef]

43. Schenk, T. RF Imperfections in High-Rate Wireless Systems; Springer: Berlin, Germany, 2008.

44. Maham, B.; Tirkkonen, O.; Hjorungnes, A. Impact of Transceiver I/Q Imbalance on Transmit Diversity of Beamforming OFDM Systems. IEEE Trans. Commun. 2012, 60, 643-648.

45. Boulogeorgos, A.A.; Sofotasios, P.C.; Selim, B.; Muhaidat, S. Effects of RF Impairments in Communications Over Cascaded Fading Channels. IEEE Trans. Veh. Technol. 2016, 65, 8878-8894. [CrossRef]

46. Qi, J.; A ̈̈ssa, S.; Alouini, M. Impact of I/Q imbalance on the performance of two-way CSI-assisted AF relaying. In Proceedings of the 2013 IEEE Wireless Communications and Networking Conference (WCNC), Shanghai, China, 7-10 April 2013; pp. 2507-2512. [CrossRef]

47. Li, X.; Liu, M.; Deng, D. Power Beacon Assisted Wireless Power Cooperative Relaying using NOMA with Hardware Impairments and Imperfect CSI. AEU-Int. J. Electron. Commun. 2019, 108, 275-286.

48. Li, X.; Li, J.; Liu, Y.; Ding, Z. Outage Performance of Cooperative NOMA Networks with Hardware Impairments. In Proceedings of the 2018 IEEE Global Communication Conference, Abu Dhabi, UAE, 9-13 December 2018; pp. 1-6. [CrossRef]

49. Aloqaily, M.; Balasubramanian, V.; Zaman, F.; Al Ridhawi, I.; Jararweh, Y. Congestion mitigation in densely crowded environments for augmenting qos in vehicular clouds. In Proceedings of the 8th Symposium on Design and Analysis of Intelligent Vehicular Networks and Applications, Montreal, QC, Canada, 28 October-2 November 2018; pp. 49-56.

(c) 2019 by the authors. Licensee MDPI, Basel, Switzerland. This article is an open access article distributed under the terms and conditions of the Creative Commons Attribution (CC BY) license (http://creativecommons.org/licenses/by/4.0/). 